\title{
Peptide Nucleic Acid Clamping
}

National Cancer Institute

\section{Source}

National Cancer Institute. Peptide Nucleic Acid Clamping. NCI Thesaurus. Code C99487.

A modification of the standard polymerase chain reaction that increases the ability to preferentially amplify mutant alleles in the presence of an excess of wild-type molecules. Wild-type molecules are rendered inactive for amplification by binding of a peptide nucleic acid (PNA) probe that hybridizes to wild-type sequence with greater affinity and under less stringent conditions. 